\title{
Utilização da relação albumina/creatinina no diagnóstico de microalbuminúria
}

\section{Diagnostic value of the albumin/creatinine ratio in detecting microalbuminuria}

Paula Virgínia Bottini'; Suzy Helena Afaz; Sidnei Silveira'; Célia Regina Garlipp ${ }^{4}$

\section{unitermos \\ Relação albumina/creatinina}

Microalbuminúria

Urina isolada

\section{resumo}

Introdução: A análise de diversos componentes urinários apresenta ampla variação biológica. A fim de se reduzir o impacto desta variabilidade na determinação de microalbuminúria, têm-se utilizado amostras de urina de 24 horas ou de repouso, o que acarreta uma série de inconvenientes e dificuldades para o paciente. Objetivo: Avaliar o desempenho da relação albumina/creatinina em amostras isoladas de urina (MALBCREA) e compará-la com a excreção urinária de albumina (EUA). Material e métodos: Foram analisadas amostras de urina (repouso e isolada) de 97 pacientes. A determinação da albumina urinária foi realizada por nefelometria (BNII - Dade). Creatinina urinária foi dosada através da reação de Jaffé modificada (Hitachi 917 - Roche). A análise da regressão foi utilizada para avaliar a correlação entre os resultados obtidos. $O$ desempenho da relação MALBCREA no diagnóstico de microalbuminúria foi calculado considerando-se o nível de decisão de $30 \mathrm{mg} / \mathrm{g}$. Resultados: Entre os pacientes estudados, 33 apresentavam EUA alterada ( $>20 \mu \mathrm{g} / \mathrm{min}$ ). A relação MALBCREA em amostras de urina apresentou correlação altamente significativa com a EUA em amostras de repouso $(r=0,902 ; p<0,05)$. Estes resultados podem ser comparados através da equação $y=1,34 x+51,33$, onde $x=$ EUA e $y=$ MALBCREA. O desempenho da relação albumina/creatinina no diagnóstico de microalbuminúria foi: sensibilidade $=88 \%$, especificidade $=90 \%$, valor preditivo positivo $(\mathrm{VPP})=83 \%$, valor preditivo negativo $(\mathrm{VPN})=$ $93 \%$ e eficiência $=90 \%$. Conclusão: Nossos resultados demonstram a validade de se utilizar a relação albumina/creatinina em amostras de urina para o diagnóstico de microalbuminúria. abstract

Introduction: Urine analysis is affected by the great biological variability for most analytes. Microalbuminuria determination usually requires an overnight or 24-hour urine collection, which can be inconvenient because of its heavy dependence on patient compliance and is frequently unreliable because of errors in collecting the required urine sample. Objective: In this study, we compared the albumin urinary excretion in overnight urine collection and random samples. Material and methods: Ninety-seven patients were evaluated; overnight and second morning samples were analyzed. Urinary albumin and creatinine concentrations were determined by nephelometry (BNII - Dade) and by modified Jaffé method (Hitachi 917 - Roche), respectively. Simple linear regression (least square method) was used to evaluate the correlation between both methods. The accuracy of the albumin to creatinine ratio $(A C R)$ was assessed considering the decision level of $30 \mathrm{mg} / \mathrm{g}$. Results: 33 patients had abnormal urinary albumin excretion (UAE $>20 \mu \mathrm{g} / \mathrm{min})$. There was a significant correlation between UAE and ACR $(r=0.902, p<$ 0.05). The ACR accuracy was $90 \%$, with sensitivity of $88 \%$ and specificity of $90 \%$. Conclusion: This study shows the diagnostic value of the albumin/creatinine ratio in detecting microalbuminuria.

\section{key words}

Albumin/creatinine ratio

Microalbuminuria

Random urine sample

1. Médica patologista clínica; supervisora do Serviço de Líquidos Biológicos da Divisão de Patologia Clínica do Hospital das Clínicas da Universidade Estadual de Campinas (HC/UNICAMP).

2. Farmacêutica do Serviço de Líquidos Biológicos da Divisão de Patologia Clínica do HC/UNICAMP.

3. Técnico de laboratório do Serviço de Líquidos Biológicos da Divisão de Patologia Clínica do HC/UNICAMP.

4. Professora associada do Departamento de Patologia Clínica da Faculdade de Ciências Médicas da UNICAMP.

Trabalho apresentado no 38 Congresso Brasileiro de Patologia Clínica, Florianópolis (SC), de 22 a 25 de setembro de 2004 


\section{Introdução}

A microalbuminúria foi inicialmente observada em pacientes diabéticos insulino-dependentes e definida como sendo a excreção urinária de pequenas quantidades de albumina, insuficientes para serem detectadas pelos métodos habituais de dosagem de proteínas ${ }^{(35)}$, ou seja, uma elevação subclínica da excreção urinária de albumina. Quantitativamente, a definição de microalbuminúria varia com o tipo de amostra coletada e com a forma de expressão dos resultados.

Considera-se microalbuminúria a elevação persistente da excreção urinária de albumina entre 20 e $200 \mu \mathrm{g} / \mathrm{min}$ em amostras minutadas de repouso (overnight) ou entre 30 e $300 \mathrm{mg} / 24$ horas em amostras de urina de 24 horas $^{(4)}$. Alternativamente podem-se adotar valores de relação albu$\mathrm{mina} /$ creatinina em amostras isoladas entre 30 e $300 \mathrm{mg} / \mathrm{g}$ ou 2,5 a $25 \mathrm{mg} / \mathrm{mmol}^{(2,14)}$.

Apesar de ser considerada um marcador precoce de lesão glomerular em indivíduos diabéticos, a microalbuminúria também ocorre em indivíduos não-diabéticos, especialmente em casos de hipertensão arterial sistêmica (HAS) e em associação com doenças cardiovasculares ${ }^{(16,31)}$, e recentemente tem sido considerada um novo fator de risco para aterosclerose ${ }^{(21,36)}$. Desta forma, a medida precisa e acurada da excreção urinária de albumina reveste-se de grande importância clínica.

A análise de diversos componentes urinários apresenta ampla variação biológica, podendo exceder 50\% em muitos $\operatorname{casos}^{(33)}$. Com relação à excreção urinária de albumina, vários estudos têm relatado uma variabilidade intra-individual, com coeficiente de variação de até $162 \%$ em pacientes diabéticos e não-diabéticos ${ }^{(9,15,22,27,28)}$. A fim de se reduzir o impacto desta variabilidade na determinação de microalbuminúria, têm-se utilizado amostras de urina de 24 horas ou de repouso. Entretanto, este tipo de coleta acarreta uma série de inconvenientes e dificuldades para o paciente, levando a inúmeros erros ${ }^{(20)}$.

A escolha da amostra ideal para dosagem de microalbuminúria ainda é controversa, embora vários estudos tenham sugerido que a determinação da relação albumina/creatinina em amostras isoladas seja comparável com os resultados obtidos em amostras minutadas $(3,6,17)$.

\section{Objetivo}

O objetivo deste estudo foi avaliar o desempenho da relação albumina/creatinina em amostras isoladas de urina (MALBCREA) e compará-la com a excreção urinária de albumina (EUA).

\section{Material e método}

\section{Casuística}

Foram estudados 97 pacientes ( 46 homens e 51 mulheres) com ampla faixa de excreção urinária de proteínas. A faixa etária variou de 6 a 88 anos (média de 47 anos). A indicação para a realização do exame incluiu diabetes melittus $(n=63)$, exposição crônica a agrotóxicos ou metais pesados $(n=11)$, avaliação de potencial doador renal $(n=8)$, cardiopatia isquêmica ou hipertensiva $(n=7)$ e outras condições $(n=8)$.

Para cada paciente foram analisadas uma amostra de urina de repouso e uma amostra isolada. A amostra isolada foi recolhida após o término da coleta da urina de repouso, representando a segunda micção da manhã. Para a coleta de urina de repouso o paciente foi orientado a esvaziar a bexiga antes de deitar e, ao levantar, coletar todo o volume urinário, anotando os horários em que foi dormir e em que se levantou.

\section{Métodos}

A determinação da albumina urinária foi realizada por nefelometria (BNII - Dade). A creatinina urinária foi dosada através da reação de Jaffé modificada (Hitachi 917 - Roche). Os resultados foram expressos como excreção de albumina por minuto $(\mu \mathrm{g} / \mathrm{min})$ e em relação à creatinina $(\mathrm{mg}$ de albumina/g de creatinina).

\section{Análise estatística}

A análise da regressão foi utilizada para avaliar a correlação entre os resultados obtidos. $O$ desempenho da relação albumina/creatinina no diagnóstico de microalbuminúria foi calculado considerando-se o nível de decisão de $30 \mathrm{mg} / \mathrm{g}$, através da construção de uma tabela $2 \times 2$ e posterior análise pelo qui-quadrado. $O$ nível de significância foi definido como $\alpha=0,05$.

\section{Resultados}

A excreção urinária de albumina variou de zero a 3.187 $\mu \mathrm{g} /$ min, sendo que a relação albumina/creatinina variou de zero a $5.377 \mathrm{mg} / \mathrm{g}$. Entre os pacientes estudados, 33 apresentavam excreção urinária de albumina alterada (>20 $\mathrm{g} / \mathrm{min})$.

A relação MALBCREA em amostras de urina apresentou correlação altamente significativa com a EUA em amostras de repouso $(r=0,902 ; p<0,05)$. Estes resultados podem ser comparados através da equação $y=1,34 x+51,33$, onde $x$ = EUA e $y=$ MALBCREA (Figura). 


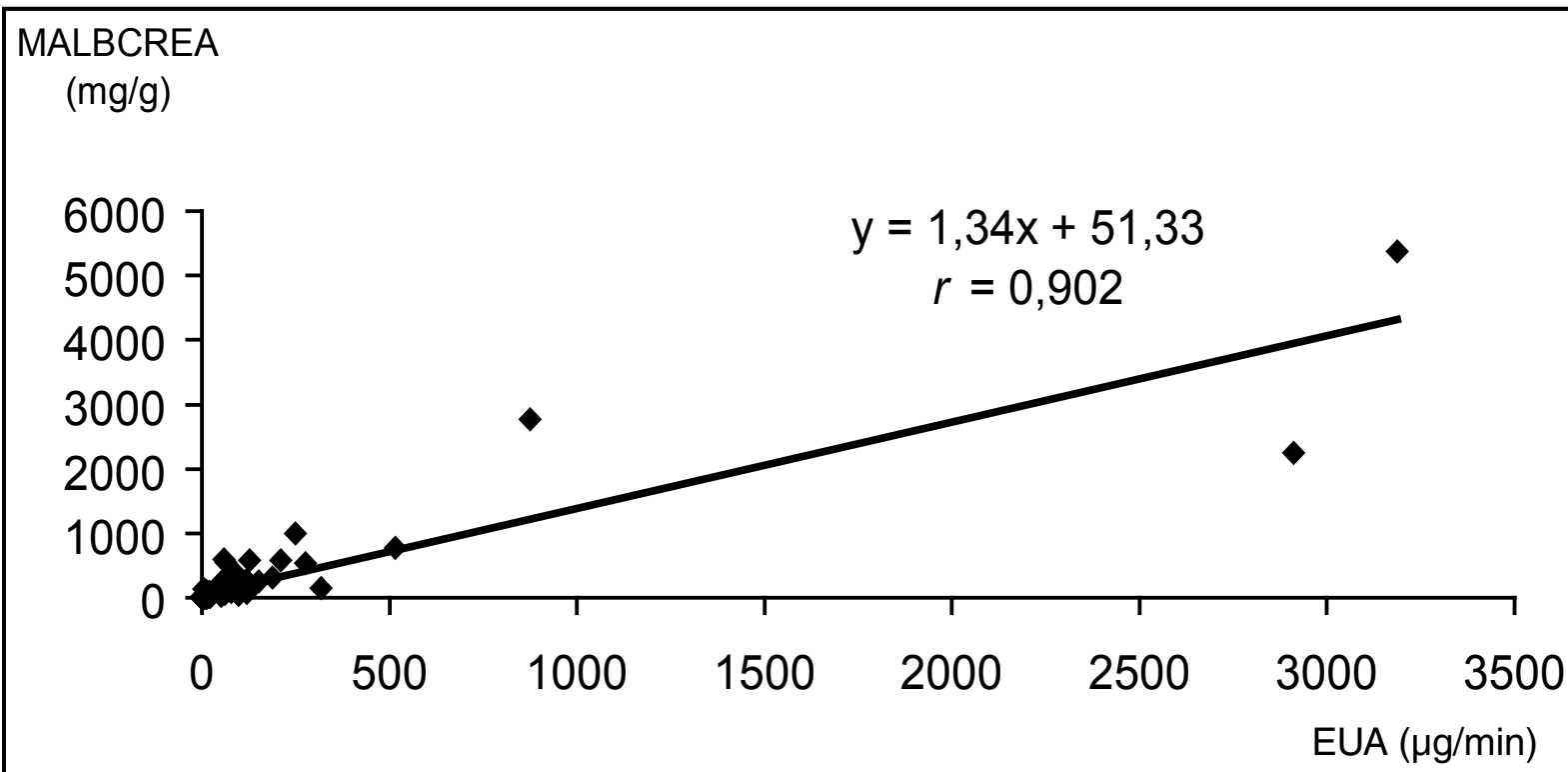

Figura - Relação entre excreção urinária de albumina e relação albumina/creatinina

O desempenho da relação albumina/creatinina no diagnóstico de microalbuminúria foi: sensibilidade $=88 \%$, especificidade $=90 \%$, valor preditivo positivo (VPP) $=83 \%$, valor preditivo negativo (VPN) $=93 \%$ e eficiência $=90 \%$ $\left(\chi^{2}=116,4 ; p<0,05\right)$ (Tabela).

\section{Discussão}

Os estudos iniciais sobre o papel da microalbuminúria como fator de risco para nefropatia diabética e doença microvascular foram realizados em amostras de urina de 24 horas ou de repouso ${ }^{(23,35)}$, uma vez que existe grande variabilidade intra-individual na taxa de excreção de albumina. Esta variabilidade pode ser causada por infecções do trato urinário, exercícios físicos, ingestão de proteínas, fatores hemodinâmicos e ritmo circadiano no transporte glomerular de macromoléculas ${ }^{(18,19)}$. Outros fatores incluem gravidez, doenças agudas e pobre controle metabólico nos indivíduos diabéticos ${ }^{(7,25)}$.

Tabela

\section{Desempenho da relação albumina/} creatinina

\begin{tabular}{lccc}
\hline & $\begin{array}{c}\text { EUA } \\
20 \mu \mathrm{g} / \mathrm{min}\end{array}$ & $\begin{array}{c}\text { EUA }< \\
20 \mu \mathrm{g} / \mathrm{min}\end{array}$ & Total \\
\cline { 1 - 3 } MALBCREA $>30 \mathrm{mg} / \mathrm{g}$ & 29 & 6 & 35 \\
MALBCREA < 30mg/g & 4 & 58 & 62 \\
Total & 33 & 64 & 97 \\
\hline$S=88 \% ; E=90 \% ; V P P=83 \% ; V P N=93 \% ;$ eficiência $=90 \%\left(\chi^{2}=116,4 ; p<0,05\right)$.
\end{tabular}

A coleta de amostras de urina de 24 horas é um procedimento bastante inconveniente na prática clínica devido a sua grande dependência do grau de colaboração e compreensão por parte do paciente, sendo freqüentemente sujeita a erros ${ }^{(8,30)}$. Um estudo realizado por Shaw et al. mostrou que $15 \%$ a $30 \%$ das amostras de urina minutadas tinham que ser rejeitadas devido a erros de coleta ${ }^{(32)}$.

Por mais de 15 anos tem-se procurado uma alternativa viável às coletas de urina minutadas. Recentemente vários autores têm demonstrado a validade da utilização da relação albumina/creatinina como método de triagem para microalbuminúria ${ }^{(1,20,29)}$. Outros estudos também mostraram uma correlação altamente significativa entre a excreção urinária de albumina em amostras minutadas (24 horas ou de repouso) e a primeira urina da manhã ${ }^{(17,24,26)}$.

No presente estudo observamos correlação significativa entre a relação albumina/creatinina em amostras isoladas de urina (segunda micção da manhã) e excreção urinária de albumina em amostras de repouso $(r=0,902$; $p<0,01)$, resultado bastante semelhante ao obtido por Assadi em comparação com amostras urina de 24 horas e segunda urina da manhã $\tilde{a}^{(3)}$. Outro estudo sugere que a relação albumina/creatinina é um bom teste de triagem para microalbuminúria, mas não deve ser utilizado como método diagnóstico por apresentar uma taxa de resultados falso-positivos que varia de $11 \%$ a $32 \%$ e que aumenta progressivamente com a idade, a partir dos 40 anos ${ }^{(13)}$.

Apesar de um relato detalhado de Koopman et al. ${ }^{(19)}$ mostrar uma variabilidade circadiana no clearance renal de 
proteínas em pacientes com síndrome nefrótica, Guder e Hofmann ${ }^{(11,12)}$, analisando os mecanismos de excreção glomerular e tubular de proteínas, demonstraram que a análise da segunda urina da manhã fornece o mesmo resultado que amostras de 24 horas, tornando desnecessária a coleta de urina de 24 horas. Da mesma forma, Ginsberg et al. ${ }^{(8)}$ sugerem a utilização da segunda urina da manhã para análise de proteínas urinárias. Estudos recentes reforçam esta tendência, evitando assim os inconvenientes e erros decorrentes da coleta de amostras de urina de 24 horas ou de repouso ${ }^{(5,34)}$.

\section{Conclusões}

Nossos resultados demonstram a validade de se utilizar a relação albumina/creatinina em amostras de urina para o diagnóstico de microalbuminúria.

\section{Referências}

I.AHN, C.W. et al.The validity of random urine specimen albumin measurement as a screening test for diabetic nephropathy. Yonsei Med J, v. 40, n. I, p. 40-5, 1999.

2.AMERICAN DIABETES ASSOCIATION. Nephopathy in diabetes. Diabetes Care, v. 27, p. 579-83, suppl. I, 2004.

3.ASSADI, F. K. Quantification of microalbumninuria using random urine sample. Pediatr Nephrol, v. 17, n. 2, p. 107-10, 2002.

4. BAKRIS, G. I. Microalbuminuria: what is it? Why is it important? What should be done about it? I Clin Hypertens, v. 3, n. 2 , p. 99- |02, 200|

5. BOTTINI, P. V.; RIBEIRO ALVES, M. A. V. F.; GARLIPP, C. R. Electrophoretic pattern of concentrated urine: comparison between 24-hour collection and random samples. Am J Kidney Dis, v. 39, n. I, p. I- 4, 2002.

6. DERHASCHNIG, U. et al. Microalbumin measurement alone or calculation of albumin/creatinine ratio for the screening of hypertension patients. Nephrol Dial Transplant, v. 17, p. $81-5,2002$.

7. GIAMPIETRO, O; CLERICO, A. Microalbuminuria in diabetes: which method to employ, which sample to collect. J Nucl Med Allied Sci, v. 34, p. I I- 120, 1990.

8. GINSBERG, J. M. et al. Use of single voided urine samples to estimate quantitative proteinuria. N Engl J Med, v. 309, n. 25 , p. 1543-46, 1983.

9. GOMES, M. B. et al. Microalbuminuria and associated clinical features among Brazilians with insulin dependent diabetes mellitus. Diabetes Res Clin Practice, v. 29, p. 143-7, 1997.

10. GOMES, M. B.; GONÇALVES, M. F. R. Is there a physiological variability for albumin excretion rate? Study in patients with diabetes type I and non-diabetic individuals. Clin Chem Acta, v. 304, p. I 17-23, 2001

I I. GUDER, W. G.; HOFMANN, W. New strategies in screening urine for exclusion and differentiation of renal diseases by analyzing individual proteins. Juogoslov Med Biochem, v. 16 , n. 2, p. 69-75, 1997.

12. HOFMANN,W:; GUDER,W. G.The diagnostic program for the analysis of proteinuria. J Clin Chem Clin Biochem, v. 27, n. 9, p. 589-600, 1989.

13. HOULIHAN, C.A. et al. Albumin to creatinine ratio: a screening test with limitations. Am J Kidney Dis, v. 39, n. 6, p. I I 83-9, 2002.

I4. ISPAD. Consensus Guidelines for the management of type I Diabetes Mellitus in children and adolescents, 2000. Disponível em http://www.d4pro.com/diabetesguidelines/. Acesso em: 8 nov, 2004.

15. JOHNSTON, J.; PATERNSON, K. R; O'REILLY, D. S.T. Estimating urinary albumin excretion rate of diabetic patients in clinical practice. BMJ, v. 306, p. 493-4, 1993.

I 6. KARALLIEDDE, j;VIBERTI, G. Microalbuminuria and cardiovascular risk. Am J Hypertens, v. 17, n. 10, p. 989-93, 2004.

17. KHAWALI, C.; ANDRIOLO, A.; FERREIRA, S. R. G. Comparison of methods for urinary albumin determination in patients with type I diabetes. Braz J Med Biol Res, v. 35, n. 3, p. 327 43, 2002.

18. KASSIRER, J. P.; HARRINGTON, J.T. Laboratory evaluation of renal function. In: SCHRIER, R., GOTTSCHALKS, C. W. editors. Diseases of the kidney. Boston: Little Brown, 1988. p. 393-44I, vol I.

19. KOOPMAN, M. G. et al. Circadian rhythm in glomerular transport of macromolecules through large pores and shunt pathway. Kidney Int, v. 49, p. 1242-9, 1996

20. LEPORE, G. et al. Cost-effectiveness of two screening programs for microalbuminuria in type 2 diabetes. Diabetes Care, v. 25 , n. II, p. $2103-4,2002$

2 I. MANN, J. F.; VI, Q. I.; GERSTEIN, H. C.Albuminuria as a predictor of cardiovascular and renal outcomes in people with known atherosclerotic cardiovascular disease. Kidney Int, v. 66, suppl. 92, p. S59-62, 2004

22. MATHIESEN, E. R. et al. Prevalence of microalbuminuria in children with type I (insulin-dependent) diabetes mellitus. Diabetologia, v. 29, p. 640-3, 1986.

23. MATTOCK, M. B. et al. Prospective study of microalbuminuria as predictor of mortality in NIDDM. Diabetes, v. 4I, n. 6, p. 736-4I, 1992.

24. MEINHARDT, U. et al. Microalbuminuria in diabetes mellitus: efficacy of a new screening method in comparison with timed overnight urine collection. J Diabetes Complications, v. 17, n. 5, p. 254-7, 2003

25. MOGENSEN, C. E. et al. Microalbuminuria and potential confounders. A review and some observations on variability of urinary albumin excretion. Diabetes Care, v. I8, p. 572 81,1995

26. MUNDET TUDURI, $X$. et al. Albumin to creatinine ratio as a diagnostic tool for type 2 diabetic nephropathy. Med Clin (Barc), v. II6, n. 19, p. 732-3, 2001 
27. ROWE, D. J. F.; BAGGA, H.; BETTS, P. B. Normal variation in rate of albumin to creatinine ratios in overnight and daytime urine collection in non-diabetic children. Br Med J, v. 291, p. 693-4, 1984.

28. SALARDI, S. et al. Microalbuminuria in diabetic children and adolescents. Relationship with puberty anf growth hormone. Acta Pediatr Scand, v. 79, p. 437-43, 1990.

29. SCHULTZ, C. J. et al. Risk of nephropathy can be detected before the onset of microalbuminuria during the early years after diagnosis of type I diabetes. Diabetes Care, v. 23, p. | 8||$-5,2000$.

30. SCHWAB, S.J. et al. Quantitation of proteinuria by the use of protein-to-creatinine ratios in single urine samples. Arch Inter Med, v. 147, p. 943-4, 1987.

3।. SEGURA, J.; CAMPOS, C.; RUILOPE, L. M. Effect of proteinuria and glomerular filtration rate on cardiovascular risk in essential hypertension. Kidney Int, v. 66, p. S45-9, suppl. 92, 2004.
32. SHAW, A. B.; RISDON P.; LEWIS-JACKSON, J. D. Proteincreatinine index and albustix in assessment of proteinuria. BrMed J, v. 287, p. 929-32, 1983.

33. SHIHAB, J. K.; SCHWARTZ, R. P.; PUGIA, M. J. Decreasing the variability observed in urine analysis. Ann Clin Lab Sci, v. 3I, n. I, p. 99- 102, 2001.

34. UMBREIT, A.; WIEDEMANN, G. Determination of urinary protein fractions. A comparison with different electrophoretic methods and quantitatively determined protein concentrations. Clinica Chimica Acta, v. 297, n. I-2, p. I36-72, 2000.

35. VIBERTI, G. C. et al. Microalbuminuria as a predictor of linical nephropathy in insulin-dependent diabetics. Lancet, v. I, p. 1430-2, 1982.

36. YOKOYAMA, $H$. et al. Subclinical atherosclerosis is increased in type 2 diabetic patients with microalbuminuria evaluated by intinma-media thickness and puls e wave velocity. Kidney Int, v. 66, n. I, p. 448-54, 2004. 